\title{
Circulating free methylglyoxal as a metabolic tumor biomarker in a rat colon adenocarcinoma model
}

\author{
PHILIPPE IRIGARAY ${ }^{1,2^{*}}$ and DOMINIQUE BELPOMME ${ }^{1-3^{*}}$ \\ ${ }^{1}$ Association for Research Against Cancer, F-75015 Paris, France; ${ }^{2}$ European Cancer and Environment Research Institute, \\ B-1000 Brussels, Belgium; ${ }^{3}$ Department of Cancer Clinical Research, Paris V University Hospital, F-75005 Paris, France
}

Received April 16, 2019; Accepted December 11, 2019

DOI: $10.3892 /$ mco.2020.2000

\begin{abstract}
Since the 1956 hypothesis of Otto Warburg, aerobic glycolysis has been recognized as a metabolic hallmark of cancer. Because methylglyoxal (MG) is a naturally occurring waste metabolite of glycolysis, we measured blood levels of this molecule in colon cancer-bearing rats. To compare the blood levels of free MG in cancerous and healthy animals, the present study used a dedicated tumor graft model consisting of the subcutaneous administration in syngenic BD-IX rats of a tumorigenic cell clone (PROb) and another non-tumorigenic clone (REGb) derived from the same tumor. Rats grafted with the PROb growing tumor cell clone exhibited a statistically significant increase in free MG blood levels $(\mathrm{P}=0.003)$, whereas rats transplanted with the REGb non-growing tumor cell clone exhibited normal MG values. The present study (first of three parts) suggests that cancer cells can produce and release free $\mathrm{MG}$ at higher levels than normal cells, making MG a putative novel metabolic biomarker of cancer.
\end{abstract}

\section{Introduction}

With the growing incidence of cancer worldwide and the persisting high number of deaths due to late discovery of the disease, the identification of new reliable cancer biomarkers is urgently needed for early detection, for follow-up of patients, and for targeted therapeutic interventions to achieve prevention and better outcomes $(1,2)$. A major challenge in harnessing the discovery of new cancer biomarkers is that cancer initiation and promotion and tumor progression are indeed complex processes involving various abnormal genetic and epigenetic

Correspondence to: Professor Dominique Belpomme, Association for Research Against Cancer, 57/59 Rue de la Convention, F-75015 Paris, France

E-mail: contact.belpomme@gmail.com

${ }^{*}$ Contributed equally

Key words: methylglyoxal, tumor biomarker, cancer metabolism, aerobic glycolysis, colon cancer molecular mechanisms and cellular interactions (3). Moreover, these processes may result from exposure to multiple and diverse environmental carcinogenic agents in different susceptible hosts. Such complexity accounts for the fact that cancers vary widely in etiology and pathogenesis (4), placing a premium on the discovery of new reliable biomarkers of malignant tumors.

Since Otto Warburg published in 1956 his hypothesis on the aerobic glycolysis of cancer cells (5), it was supposed that to generate ATP, malignant cells reduce mitochondrial respiration and compensate it by increasing glycolysis and even can replace it totally by glycolysis, despite sufficient amounts of oxygen penetrating into malignant cells (6). Aerobic glycolysis was thus recognized as a putative hallmark of cancer cell metabolism (7); and today's hypothesis is that to promote cancer cell survival and proliferation, suppressing mitochondrial respiration may increase glycolysis for ATP production, whereas maintaining mitochondrial respiration puts cancer cells at risk of apoptosis (8). By bypassing the Embden-Meyerhof-Parnas glycolytic pathway (9), a key waste glycolysis-associated metabolite preceding formation of the end product D-Lactate is the aldehyde form of pyruvic acid, methylglyoxal (MG), also called pyruvaldehyde or 2-oxopropanal $\left(\mathrm{CH}_{3}-\mathrm{CO}-\mathrm{CH}=\mathrm{O}\right.$ or $\left.\mathrm{C}_{3} \mathrm{H}_{4} \mathrm{O}_{2}\right)(10)$.

Because aerobic glycolysis appears to be a general metabolic property of malignant cells and because MG is a naturally occurring metabolic byproduct of glycolysis, we measured levels of this molecule in the peripheral blood of rats using a specially dedicated tumor graft experimental model, to determine whether free MG can be a reliable tumor growth-associated biomarker. This paper is the first part of a recently patented three step study showing that free MG is a reliable metabolic biomarker of cancer growth (11).

\section{Materials and methods}

Experimental design. To compare the peripheral blood levels of free MG in cancerous and healthy animals we used a DHD non-diabetic rat colonic adenocarcinoma tumor model from which two cell clones were derived, one being tumorigenic (PROb) and the other non-tumorigenic (REGb), both obtained from a colonic adenocarcinoma in BD-IX rats. As previously reported, the tumor progressive PROb (DHD-K12/TRb) clone and the tumor regressive REGb (DHD-K12/TSb) clone 
were established from a unique DHD colon adenocarcinoma induced by 1-2 dimethylhydrazine in a BD-IX strain of female rat (12).

Animals and tumor growth assay. Syngeneic BD-IX rats aged 8-10 weeks were used in the tumor growth assay. BD-IX rats initially provided by Charles River were harvested locally in the animal facility (research unit U1098 animal facility (Dijon, France) of the French Institute of Health and Medical Research (INSERM). Animal health and behavior were monitored twice a week. The welfare of animals used in this assay was in agreement with international guidelines. Animals were housed under specific pathogen-free conditions and cared for according to the guidelines of the institutional Veterinary Committee and the European laws for animal experimentation. More precisely animal use and handling were approved by the local veterinary committee and were performed according to the European laws for animal experimentation including the use of anesthetics and efforts to minimize animal distress. For anesthesia, we used isoflurane. We placed animals in an induction chamber and adjusted the oxygen flowmeter to $0.8-1.5 \mathrm{l} / \mathrm{min}$ and the isoflurane vaporizer to $3-5 \%$. After prolonged exposure on isoflurane, cervical dislocation were performed. During the 9 week duration of the experiments, we observed no spontaneous death and all animals being euthanized at the end of the experimental period.Cells of the two clones were cultured in Ham F10 medium (BioWhittaker) complemented with $10 \%$ fetal bovine serum (Gibco; Thermo Fisher Scientific, Inc.). Cells $\left(1 \times 10^{6}\right)$ of the two clones were injected subcutaneously in $100 \mu 1$ of serum-free Ham F10 medium into the antero-lateral thoracic wall of BD-IX rats.

Blood samples were harvested at 0, 2, 3, 4, 6 and 9 weeks after the cell injection of each clones in 18 animals ( 9 males and 9 females). Blood (3-5 ml) was collected through orbital cavity or $5 \mathrm{ml}$ by cardiac puncture after rats were sacrificed by cervical dislocation.

Tumor volume was measured, using a caliper to determine two perpendicular diameters. We calculated tumor volume (V) from the caliper measurements using the formula $V=\left(W^{2} x L\right) / 2$, where $\mathrm{W}$ is the tumor width and $\mathrm{L}$, the tumor length.

The endpoint assessment was based on tumor volume measurement according to the pre-established time scale i.e., at $0,2,3,4,6$ and 9 weeks during which blood samples were obtained.

During the experimental process all animals were checked twice a week for suffering analysis which include particularly prostration, damaged fur, half-closed eyes and irritated eyes. We never observed any suffering of the animal so we did not stop the experimental process up to week 9 (T9). Tumor volume never exceeded $3,000 \mathrm{~mm}^{3}$ at $\mathrm{T} 9$.

\section{MG measurement}

Chemicals and technical devices used in the assay. All chemicals were analytical grade. MG (40\% aqueous solution), glyoxalase I (from Saccharomyces cerevisiae, grade IV, buffered aqueous glycerol solution) and reduced glutathione (GSH) were supplied by Sigma-Aldrich, Merck KGaA. O-phenylenediamine also called 1,2-diaminobenzene (O-PD) was purchased from AcrosOrganics-Fisher Scientific and 5-methylquinoxaline (5-MQX) from Interchim. Acetonitrile and trifluoroacetic acid (TFA) for High performance liquid chromatography (HPLC) gradient grade were purchased from Carlo Erba and formic acid (99-100\%, Normapur) from Grosseron. Water used in these experiments was purified using a Milli-Q Water Purification System (EMD Millipore Corp.). The reversed phase chromatography column (symmetry C18: $3.9 \times 150 \mathrm{~mm} ; 5 \mu \mathrm{m})$ and the Symmetry Sentry ${ }^{\mathrm{TM}}$ guard column (3.9x20 mm, $5 \mu \mathrm{m})$ were purchased from Waters (Milford).

Preparation of blood samples. The whole blood $(0.5 \mathrm{~g})$ was diluted twice in water and the proteins were precipitated with TFA (50 $\mu 1,0.65 \mathrm{M}$ final concentration). The supernatant obtained by centrifugation $\left(12,000 \mathrm{~g}, 10 \mathrm{~min}, 4^{\circ} \mathrm{C}\right)$ was supplemented with the deriviatising agent, o-PD $(50 \mu \mathrm{l}$ of a $0.01 \%$ solution in water) and incubated at $23^{\circ} \mathrm{C}$ for $6 \mathrm{~h}$ in the dark. After centrifugation $(10,000 \mathrm{~g}, 5 \mathrm{~min})$ the supernatant was then analysed by using an HPLC for quantitative analysis of the 2-quinoxaline free MG derivative (2-MQX) by liquid chromatography-electrospray ionization (ESI) mass spectrometry (MS) (ESI-MS) method with 5-MQX used as internal standard.

Preparation of standards. The concentration of the stock aqueous solution of free MG was determined enzymatically by endpoint assay. Free MG quantification involves conversion to S-D-lactoylglutathione by glyoxalase I in the presence of GSH (Fig. 1). Calibrating standards containing 0.0625-1.6 $\mu \mathrm{mol}$ of $\mathrm{MG}$ in $1 \mathrm{ml}$ of water were prepared. Derivatization was carried out by the procedure described above (see 'preparation of blood sample'). Calibration curves were constructed by plotting the pick area ratios of 2-MQX and 5-MQX internal standard against the MG concentrations.

MG dosage. MG was measured in the whole blood using an already described method (11). To identify free MG and quantify its blood level in studied animals, we used a free MG dosage method adapted from that of Chaplen et al (13) and that of Rabbani and Thornalley (14) but using whole blood instead of plasma, and TFA instead of trichloroacetic acid as precipitating agent. We also did not add sodium azide because according to the method we used we did not find any statistically significant difference with or without this anti-peroxidase agent. In any case our results were established in comparison with normal T0 values.

The quinoxaline derivatives (2-MQX and 5-MQX) were resolved by reversed-phase HPLC (RP-HPLC) and analysed by ESI/single ion monitoring.

Chromatographic analysis was performed using a Waters Alliance e2695 separation module (Waters) coupled with a Waters Micromass ZQ 2000 module (Waters) as ESI-MS Detector. The analytical column employed was a Symmetry C18 analytical column $100 \AA$, $5 \mu \mathrm{m}, 3.9 \times 150 \mathrm{~mm}$ combined with a Symmetry Sentry TM guard column $100 \AA, 5 \mu \mathrm{m}$, $3.9 \times 20 \mathrm{~mm}$ from Waters. The mobile phase consisted of solvent A: $0.5 \%(\mathrm{v} / \mathrm{v})$ formic acid in water and solvent $\mathrm{B}$ : $0.5 \%(\mathrm{v} / \mathrm{v})$ formic acid in acetonitrile, and was delivered at $0.8 \mathrm{ml} / \mathrm{min}$. The program of HPLC run was the following: $12-18 \%$ of mobile phase B over $4 \mathrm{~min}$ and held for $16 \mathrm{~min}$. This was followed by $18 \%$ solvent B for $16 \mathrm{~min}$. 


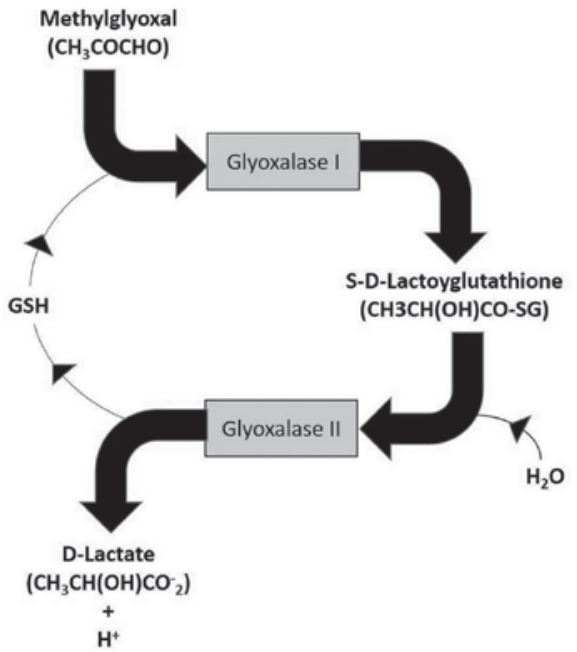

Figure 1. Role of the glyoxalase I and II system in the intracellular detoxification of methylglyoxal. GSH, glutathione.

The injection volume was $85 \mu \mathrm{l}$ of the sample and $5 \mu \mathrm{l}$ of a 5-MQX water solution $(1 \mathrm{mg} / \mathrm{ml}$ diluted $1 / 200)$. The autosampler temperature was maintained at $4^{\circ} \mathrm{C}$ throughout the analysis.

The quinoxaline derivatives (2-MQX and 5-MQX) were resolved by reversed-phase HPLC (RP-HPLC) and analysed by ESI/single ion monitoring.

MS conditions were as follows: Cone voltage, $30 \mathrm{~V}$; source temperature, $110^{\circ} \mathrm{C}$; desolvatation gas flow, $400 \mathrm{l} / \mathrm{h}$; probe temperature, $110^{\circ} \mathrm{C}$. The MS used positive ion electrospray with capillary voltage held at very high voltage (order of $\mathrm{kV}$ ). Empower software (Waters) was used to control the analytical system and data processing.

Free MG quantification was performed by calculating a peak area ratio of protonated molecular ion peak intensity $(\mathrm{m} / \mathrm{z} 145)$ to a protonated molecular internal standard ion peak intensity (5-MQX, m/z 145) in the selected ion monitoring mode.

MG measurements were done in triplicate for each blood sample analyzed and histograms were constructed by plotting each MG mean values against time for the two studied group of rats.

Statistical analysis. We used two different statistical tests: The Friedman's test with Nemenyi post-hoc comparisons concerning MG, tumoral volume and glucose concentration analysis as we hypothesized our data would not be a normal distribution for a within-group comparison between the different values obtainedat time measurement (at T0 and after 2, 3, 4, 6 and 9 weeks); and the Pearson product-moment correlation test, to measure the strength of association. All statistical analysis were conducted using the XLSTAT software. $\mathrm{P}<0.05$ was considered to indicate a statistically significant difference.

\section{Results}

Tumor development. The results are depicted in Figs. 2 and 3. Fig. 1 presents the increase in free MG blood levels and tumor volume (+/-SD) after PROb cell transplantation. As indicated

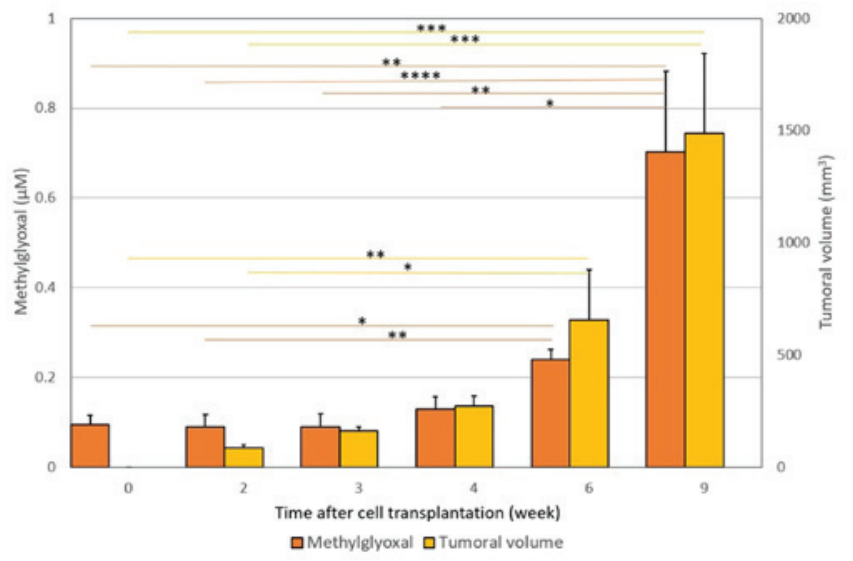

Figure 2. Increase in free MG blood levels and tumor volume $( \pm$ SD) following PROb cell transplantation. Subcutaneous injection of PROb cells into syngeneic BD-IX rats allows the establishment of progressively growing tumors. A significant 7-fold increase $(\mathrm{P}=0.003)$ in $\mathrm{MG}$ blood level was observed. Free MG blood levels increased from $0.095 \pm 0.021 \mu \mathrm{M}$ at T0, to $0.703 \pm 0.178 \mu \mathrm{M}$ at 9 weeks after transplantation. At each time point, the measured tumor volume and the MG blood level obtained at time $\mathrm{T}$ were compared with those obtained previously, i.e. T9 vs. T6, T6 vs. T4, T4 vs. T3, $\mathrm{T} 3$ vs. T2 and T2 vs. T0. Horizontal bars represent the significant statistical results obtained using Friedman's test with Nemenyi post-hoc comparisons. ${ }^{*} \mathrm{P}<0.05,{ }^{* *} \mathrm{P}<0.01,{ }^{* * *} \mathrm{P}<0.001,{ }^{* * * *} \mathrm{P}<0.0001$, as indicated. PROb cells, tumorigenic clone cells; MG, methylglyoxal.

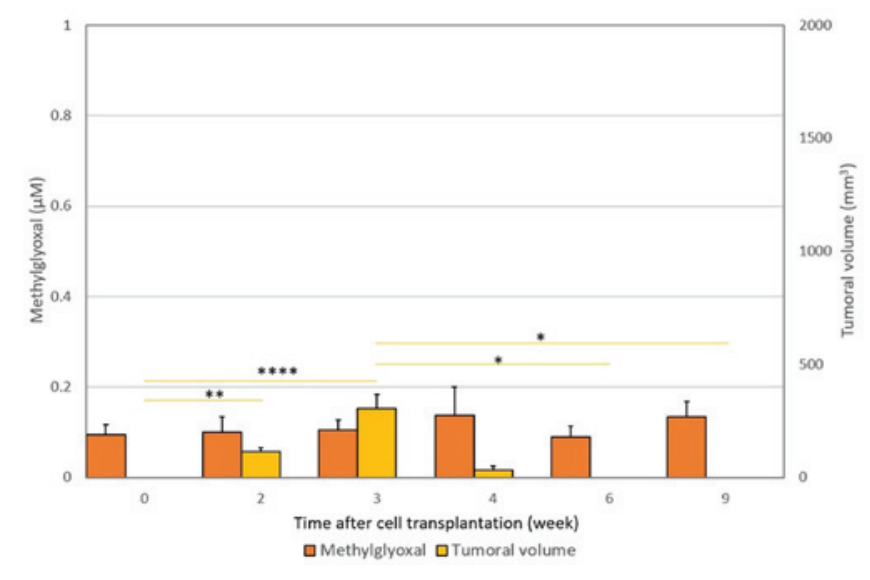

Figure 3. Lack of change in free MG blood levels and tumor volume $( \pm \mathrm{SD})$ after REGb cell transplantation. Subcutaneous injection of REGb cells into syngeneic BD-IX rats resulted in complete rejection of measurable tumors at 4 weeks after transplantation. The levels of free MG did not differ significantly from normal T0 values during the entire experimental study period $(\mathrm{P}=0.63)$. At each time point, the measured tumor volume and the MG blood level obtained at time $\mathrm{T}$ were compared with those obtained previously, i.e., T9 vs. T6, T6 vs. T4, T4 vs. T3, T3 vs. T2 and T2 vs. T0. Horizontal bars represent the significant statistical results obtained using the Friedman's test with Nemenyi post-hoc comparisons. ${ }^{*} \mathrm{P}<0.05,{ }^{* *} \mathrm{P}<0.01,{ }^{* * * * *} \mathrm{P}<0.0001$, as indicated. MG, methylglyoxal; REGb cells, non-tumorigenic clone cells.

in Fig. 2, subcutaneous injection of PROb cells into syngeneic hosts allowed the establishment of progressively growing cancerous tumors, whereas injection of REGb cells into these syngeneic hosts induced a limited and transient development of tumors which completely regressed in less than 4 weeks (Fig. 3).

Free MG blood levels. The results are depicted in Figs. 2 and 3. Rats having been grafted with PROb cells had growing tumors and a statistically significant increase in free MG 
Table I. Glucose measurement in blood of rats with progressive tumor (PROb clone).

\begin{tabular}{lccccccr}
\hline & \multicolumn{7}{c}{ Glucose $(\mathrm{mmol} / \mathrm{l}) \pm \mathrm{SD}$} \\
\cline { 2 - 8 } Sex & T0 & T4 & P-value & T6 & P-value $^{\mathrm{b}}$ & T9 & P-value $^{\mathrm{c}}$ \\
\hline Male (n=9) & $11.58 \pm 1.42$ & $13.23 \pm 1.17$ & 0.82 & $11.50 \pm 0.98$ & 0.97 & $10.67 \pm 1.63$ & 0.87 \\
Female (n=9) & $11.51 \pm 0.66$ & $14.35 \pm 1.68$ & 0.27 & $11.99 \pm 1.73$ & 0.85 & $11.86 \pm 1.23$ & 0.82 \\
Total $(\mathrm{n}=18)$ & $11.54 \pm 0.62$ & $13.74 \pm 1.06$ & 0.17 & $11.75 \pm 0.98$ & 0.92 & $11.26 \pm 1.00$ & 0.93 \\
\hline
\end{tabular}

${ }^{\mathrm{a}} \mathrm{T} 4$ vs. T0; ${ }^{\mathrm{b}} \mathrm{T} 6$ vs. T0; ${ }^{\mathrm{c}} \mathrm{T} 9$ vs. T0.

blood levels in comparison with $\mathrm{T} 0$ values $(\mathrm{P}=0.003)$, whereas rats transplanted with REGb cells were associated with tumor rejection and no statistically significant increase in free $\mathrm{MG}$ blood levels ( $\mathrm{P}=0.863)$ (Figs. 2 and 3). For the rats grafted with the growing tumor-associated PROb cells, MG levels varied from $0.095 \pm 0.021 \mu \mathrm{M}$ at time T0 to $0.703 \pm 0.178 \mu \mathrm{M}$ at week 9 after tumor transplantation (Fig. 2). Also the Pearson correlation statistical test showed a good statistical correlation between free MG blood levels and tumour volume along with tumor progression $(\mathrm{P}=0.0006)$.

Glucose blood levels. The results are depicted in Table I. It is worthy of note that during the whole study period glucose concentrationremained normal and that there was no statistical correlation between free MG levels and glucose concentration in the blood with the Pearson correlation statistical test $(\mathrm{P}=0.476)$.

\section{Discussion}

We used an already described free MG dosage method (11) adapted from Chaplen et al (13) and Rabbani and Thornalley (14) but using whole blood instead of plasma, and TFA instead of trichloroacetic acid as precipitating agent. We also did not add sodium azide because according to the method we used we did not find any statistically significant difference with or without this anti-peroxidase agent. In any case our results are presented in comparison with normal $\mathrm{T} 0$ values.

We showed that after grafting BD-IX rats with PROb tumorigenic cancer cells, there was a clear statistically significant increase in free MG blood levels and a statistically positive correlation between free MG blood levels and tumor volume; whereas for rats transplanted with cells of the REGb non-growing tumor-associated clone, for which the tumor graft did not take, there was a low free MG blood levels not differing statistically from normal levels.

In this experimental study we have showed for the first time that significantly increased blood levels of free $\mathrm{MG}$ are associated with colon cancer progression. Although it has been established that cancer development may be associated with the increased formation of MG-derived intracellular advanced glycation end-products (AGE) $(15,16)$, only one publication was specifically devoted to the measurement of MG in cancer animals (17); and more recently, only one in cancer patients, in which it was shown that MG may have cancer promoting properties (18). We emphasize that there was no previous reported study on free MG level measurement in cancer animals or cancer patients. By contrast, contrary to what we showed in this paper, it has been claimed that high dose external MG could inhibit cancer cell growth in vitro (19), a finding which has never been confirmed clinically. These different considerations may explain why the results we obtained in the present study and those we provided in vitro by studying MG production and release by cancer cells in cell culture (part 2 of our general study) and in vivo, by showing that free MG can be clinically used as a cancer biomarker (part 3 of our general study) have been considered as new data and so have been patented (11).

A major point to discuss in our study deals with the fact that free MG levels may have been increased through a putative induction of hyperglycemia since it has been shown that free MG levels can also be increased in the case of human type 2 diabetes (20). Since we used a non-diabetic rat model, this excludes a priori the possibility that the observed increase in free MG blood levels was due to diabetes (21). However since hyperglycemia have been shown to be a risk factor for cancer progression (22), we systematically measured glucose in addition to MG in the blood of rats. As indicated in Table I, glucose concentration in rats with progressive tumors (clone PROb) remained normal during the whole study period, a finding proving that the increase in free MG was not caused by the existence of a tumor progression-associated hyperglycemia. In the present study we did not perform any molecular investigation of cancer cells nor any immunohistochemistry study in tumor cell sections in an attempt to prove that free MG really are produced by cancer cells. Such a lack constitutes indeed some limitation of the hypothesis we propose that free MG can be directly produced and released by cancer cells. However such hypothesis has been validated in part 2 and 3 of our three step general study (11) by showing that cancer cell extracts contain a statistically significant higher quantity of free $\mathrm{MG}$ in comparison with that of non-cancer cells, a finding that has been demonstrated not only in cell cultures but also clinically in patients (11). In the present study, as free MG blood levels increase as the tumor progresses, and free MG blood levels remain at normal values in the case of tumor rejection by inflammatory and/or immune response, we therefore hypothesized that free MG blood levels correlate positively with the number of cancer cells in the tumor. Consequently, our data strongly suggest that levels of circulating free MG may accurately reflect the metabolic and proliferative capacity of cancer cells. An explanation could be that MG is normally detoxified by the GLO-I and GLO-II glyoxalase system up to a threshold 
concentration (23) (Fig. 1). Although GLO-I activity has been shown to be increased in many human cancers $(17,24-27)$ and particularly in aggressive tumors $(24,25)$, GLO-II activity is generally lower $(26,27)$. This means that in comparison with normal cells, cancer cells may be less capable of detoxifying intracellular free MG and so be less capable of recovering normal GSH concentration (Fig. 1). This could explain why an increase of both carbonyl stress and oxidative stress (28) is associated with tumor promotion and progression (29); and why, depending on the free radical concentration in cancer cells (28), there is an increased degree of intra-tumoral apoptosis/necrosis as the tumor progresses (7). A further limitation of our study is that we could not measure any cancer blood marker to determine precisely the growth of the tumor, due to the fact that to our knowledge such cancer marker has not been previously identified in this rats model. However since MG is a cancer-unique yet ubiquitous glycolysis-associated molecule that is produced and released from many different types of cells, including microorganisms (30) and mammalian cells (31), we propose that the experimental data obtained from this rat colon cancer model may be extended to other cancer types, and more particularly to human cancers. This is what our forthcoming clinical data (part 3 of our general study) will prove by showing that $\mathrm{MG}$ is a putative new metabolic biomarker of malignant tumors that could be used routinely for early detection and follow-up of patients and for therapeutic decision making (11).

\section{Acknowledgements}

The authors would like to thank Dr. Bernard Bonnotte, Ms. Malika Trad and Ms. Jennifer Fraszczak from CHU Dijon-Bocage, Médecine interne et Immunologie Clinique (Dijon, France) for the high quality of the animals experiments, Dr. Sylvie Barbier and Mr. Clément Poletti from Bioavenir Laboratory (Metz, France) for the high quality of the blood analysis, and Mr. Tony Tweedale from R.I.S.K. (RebuttingIndustry Science with Knowledge; Brussels, Belgium) for his review and valuable comments on an early draft.

\section{Funding}

The present study received a grant from the ARTAC (grant no. BM2706/05).

\section{Availability of data and materials}

The datasets used and/or analyzed during the current study are available from the corresponding author on reasonable request.

\section{Authors' contributions}

DB and PI conceived and designed the analysis, contributed data or analysis tools and performed the analysis. PI collected the data. Both authors wrote, read and approved the final version of the manuscript.

\section{Ethics approval and consent to participate}

Parts of the study using animals were performed at the animal facility of the University Hospital of Dijon and the
Research Unit U1098 of the French Institute of Health and Medical Research (Inserm U1098) with the ethical approval of the 'Comité d'Ethique de l'ExpérimentationAnimale Grand Campus Dijon'.

\section{Patient consent for publication}

Not applicable.

\section{Competing interests}

The authors declare that they have no competing interests. This study is part of the patent PCT/EP2013/072459 (Inventors, DB and PI; Status: national phase; the fact that free methylglyoxal blood level increases with the development of the tumor is covered by this patent).

\section{References}

1. Ludwig JA and Weinstein JN: Biomarkers in cancer staging, prognosis and treatment selection. Nat Rev Cancer 5: 845-856, 2005.

2. Henry NL and Hayes DF: Cancer biomarkers. Mol Oncol 6: 140-146, 2012

3. Irigaray $P$ and Belpomme D: Basic properties and molecular mechanisms of exogenous chemical carcinogens. Carcinogenesis 31: 135-148, 2010.

4. Belpomme D, Irigaray P, Hardell L, Clapp R, Montagnier L, Epstein S and Sasco AJ: The multitude and diversity of environmental carcinogens. Environ Res 105: 414-429, 2007.

5. Warburg O: On the origin of cancer cells. Science 123: 309-314, 1956.

6. Kim A: Mitochondria in cancer energy metabolism: Culprits or bystanders? Toxicol Res 31: 323-330, 2015.

7. Hanahan D and Weinberg RA: Hallmarks of cancer: The next generation. Cell 144: 646-674, 2011.

8. Lopez J and Tait SW: Mitochondrial apoptosis: Killing cancer using the enemy within. Br J Cancer 112: 957-962, 2015.

9. Peretó J: Embden-meyerhof-parnas pathway. In: Encyclopedia of Astrobiology. Amils R, Cernicharo Quintanilla, J, Cleaves HJ, Irvine WM, Pinti D and Viso M (eds). Springer, Berlin, Heidelberg, 2014.

10. Thornalley PJ: Pharmacology of methylglyoxal: Formation, modification of proteins and nucleic acids, and enzymatic detoxification-a role in pathogenesis and antiproliferative chemotherapy. Gen Pharmacol 27: 565-573, 1996.

11. Belpomme D and Irigaray P: Methylglyoxal as a marker of cancer. Patent PCT/EP2013/072459. Filed: October 25, 2013; issued October 25, 2017.

12. Martin F, Caignard A, Jeannin JF, Leclerc A and Martin M: Selection by trypsin of two sublines of rat colon cancer cells forming progressive or regressive tumors. Int J Cancer 32: 623-627, 1983.

13. Chaplen FW, Fahl WE and Cameron DC: Detection of methylglyoxal as a degradation product of DNA and nucleic acid components treated with strong acid. Anal Biochem 236: 262-269, 1996.

14. Rabbani $\mathrm{N}$ and Thornalley PJ: Measurement of methylglyoxal by stable isotopic dilution analysis LC-MS/MS with corroborative prediction in physiological samples. Nat Protoc 9: 1969-1979, 2014.

15. Turner DP: Advanced glycation end-products: A biological consequence of lifestyle contributing to cancer disparity. Cancer Res 75: 1925-1929, 2015.

16. Schröter D and Höhn A: Role of advanced glycation end products in carcinogenesis and their therapeutic implications. Curr Pharm Des 24: 5245-5251, 2018.

17. Matsuura T, Owada K, Sano M, Saito S, Tomita I and Ikekawa T: Studies on methylglyoxal. II. Changes of methylglyoxal level accompanying the changes of glyoxalase I and II activities in mice bearing L1210 leukemia and sarcoma 180. Chem Pharm Bull 34: 2926-2930, 1986.

18. Antognelli C, Moretti S, Frosini R, Puxeddu E, Sidoni A and Talesa VN: Methylglyoxal acts as a tumor-promoting factor in anaplastic thyroid cancer. Cells 8: E547, 2017. 
19. Talukdar D, Ray S, Ray M and Das S: A brief critical overview of the biological effects of methylglyoxal and further evaluation of a methylglyoxal-based anticancer formulation in treating cancer patients. Drug Metabol Drug Interact 23: 175-210, 2008.

20. Beisswenger PJ, Howell SK, Touchette AD, Lal S and Szwergold BS: Metformin reduces systemic methylglyoxal levels in type 2 diabetes. Diabetes 48: 198-202, 1999.

21. Lapolla A, Flamini R, Dalla Vedova A, Senesi A, Reitano R, Fedele D, Basso E, Seraglia R and Traldi P: Glyoxal and methylglyoxal levels in diabetic patients: Quantitative determination by a new GC/MS method. ClinChem Lab Med 41: 1166-1173, 2003.

22. Ryu TY, Park J and Scherer PE: Hyperglycemia as a risk factor for cancer progression. Diabetes Metab J 38: 330-336, 2014.

23. Honek JF: Glyoxalase biochemistry. Biomol Concepts 6: 401-414, 2015.

24. Zhang D, Tai LK, Wong LL, Chiu LL, Sethi SK and Koay ES: Proteomic study reveals that proteins involved in metabolic and detoxification pathways are highly expressed in HER-2/neu-positive breast cancer. Mol Cell Proteomics 4 1686-1696, 2005.

25. Chiavarina B, Nokin MJ, Durieux F, Bianchi E, Turtoi A, Peulen O, Peixoto P, Irigaray P, Uchida K, Belpomme D, et al: Triple negative tumors accumulate significantly less methylglyoxal specific adducts than other human breast cancer subtypes. Oncotarget 5: 5472-5482, 2014.
26. Hutschenreuther A, Bigl M, Hemdan NY, Debebe T, Gaunitz F, and Birkenmeier G: Modulation of GLO1 expression affects malignant properties of cells. Int J Mol 17: pii: E2133, 2016.

27. Antognelli C, Baldracchini F, Talesa VN, Costantini E, Zucchi A and Mearini E: Overexpression of glyoxalase system enzymes in human kidney tumor. Cancer J 12: 222-228, 2006.

28. Kalapos MP: The tandem of free radicals and methylglyoxal. ChemBiol Interact 171: 251-271, 2008.

29. Dreher D and Junod AF: Role of oxygen free radicals in cancer development. Eur J Cancer 32A: 30-38, 1996.

30. Inoue $\mathrm{Y}$ and Kimura A: Methylglyoxal and regulation of its metabolism in microorganisms. Adv Microb Physiol 37: 177-227, 1995.

31. Kalapos MP: Methylglyoxal in living organisms: Chemistry, biochemistry, toxicology and biological implications. Toxicol Lett 110: 145-175, 1999. International (CC BY-NC-ND 4.0) License. 